\title{
DYNAMICAL BEHAVIOR OF FRACTIONAL ORDER SVIR EPIDEMIC MODEL
}

\section{AQEEL AHMAD*, NOUMAN JAVEED, MUHAMMAD FARMAN, M.O. AHMAD, AMNA HAFEEZ AND ALI RAZA}

Department of Mathematics and Statistics, The University of Lahore, Lahore, Pakistan

${ }^{*}$ Corresponding author: aqeelahmad.740@gmmail.com

\begin{abstract}
In this Paper, we proposed a fractional order SVIR epidemic model is incorporated to investigate its dynamical behavior in random environments. S, V, I, R are known as variables and these variables represent the number of susceptible, vaccinated, infected and recovered cells from viruses in the body. The Caputo fractional derivative operator of order $\alpha \epsilon(0,1]$ is employed to obtain the system of fractional differential equations. The basic reproductive number is derived for a general viral production rate which determines the local stability of the infection free equilibrium. The stability and sensitivity analysis of fractional order has been made and verify the non-negative unique solution. The solution of the time fractional model has been procured by employing Laplace Adomian decomposition method (LADM) and the accuracy of the scheme is presented by convergence analysis. Finally numerical solutions are also established to investigate the influence of system parameter on the spread of disease and which show the effect of fractional parameter on our obtained solution.
\end{abstract}

\section{INTRODUCTION}

It is evident that science subjects such as physics and chemistry are associated with mathematics. However, biology is the subject which is not usually associated with mathematics. But with the help of technology, relevant research suggests that there are quantifiable aspects of life science as well which can be measured with the help of mathematics. Mathematics plays a very crucial role in understanding and exploring the natural world in this regard. The combination of mathematics and biology gave birth to new field that is

Received 2018-10-17; accepted 2018-11-27; published 2019-03-01.

2010 Mathematics Subject Classification. 37C75,65L07.

Key words and phrases. Stability Analysis; Dynamical transmission; Caputo fractional derivative; LADM.

(c)2019 Authors retain the copyrights of their papers, and all open access articles are distributed under the terms of the Creative Commons Attribution License. 
mathematical biology. Mathematical biology is defined as a field of research and inquiry that investigates and explores mathematical representations of biological systems.

The scope of mathematics includes mathematical modeling and esoteric mathematics. The flow of work, process, predictions and outcomes can easily be measured with the help of mathematical concepts and theory. Therefore, biologists are now extremely dependent on mathematics. Mathematical modeling of biological sciences is done by many brilliant scientist [1-3]. The relationship between simple mathematical modeling involves biological system, integer order differential equations that show their dynamics and complex system which describes their changing of structure. The nonlinearity and multi-scale behaviors in mathematical modeling describe the mutual relationship between parameters [4]. In last few decades, many biological models were studied in detail by using classical derivatives, few of them are [5-9].

Mathematics has always beneficial and play important role in the rising science. Mathematics and physical sciences each had important effects on the development of each other. Mathematics has been playing a significant role in the progress of life and social sciences, and these sciences has started to influence the development of mathematics. Biomedical science is one of the most important fields of science and expected well growing future. Biology is concern with the subject of mathematician for improvement. The mostly use of mathematics in biology become more qualitative to quantitative. Mathematical biology is the study of bio informatics and its relevant fields. The earlier stages of mathematical biology were dominated by physical systems, often involving specific mathematical models of bio systems and there components or compartments. Mathematical modeling and analysis is use to recognize biology and biomedical system[10,11]. Examine the Dynamical transmission and effect of smoking in society [18]. The distinguished features of fractional differential equations are that it outlines memory and transmitted properties of numerous mathematical models. As a fact, that fractional order models are more realistic and practical than the classical integer order models. Fractional order derivative produces greater degree of freedom in these models. Arbitrary order derivatives are powerful tools for the discretion of the dynamical behavior of various biomaterial and systems $[19,20]$.

It is expected that biomedical science will be the foremost field of research in future. For the development of the health of their subject, Mathematicians should get involved in the field of biology. There are lots of examples that suggest how mathematics has benefited from physics. It is clear that if mathematicians do not take genuine interest in the field of biosciences they will miss the opportunity to be a part of the most vital and interesting scientific Enterprises of all times. Mathematical biology is an emerging, well-recognized subject. Moreover, in my opinion it is considered the most exciting modern application of mathematics. The burgeoning use of mathematics in biology is likely to happen since the field of biology became more significant. The intricacy of the biological sciences makes integrative involvement crucial. For the mathematician, biology has opened up new avenues and windows for further research. Similarly for the 
biologist, mathematical modelling offers another research tool Proportionate with new powerful laboratory proficiency. However, it will be fruitful only if it is used relevantly recognizing its limitations.

One of the important roles mathematics plays in the field of biology is the formation and creation of mathematical models. These equations, models or formulas can anticipate or illustrate natural happenings, for example behavioral patterns of an organism and measurement of population with the passage of time.

As far as mathematical models are concerned it makes easier for scientist to analyze and describe a measurable phenomenon. It has been observed that Most of the fields of medicine are also dependent upon mathematical models. Mathematical models helps the scientist especially in the frequencies of gene expression and the rates of spreading of diseases.

For the sake of argument lets suppose that a scientist is interested in studying butterfly migrations. The biologist simply entrees into that field and count a sample population of a specific area. After that, he multiplies his sample numbers by the total geographical range to find out a population estimate. He will then return to his lab and review and read other researcher's reports and literature on butterflies. He will observe their migration pattern over the span. He will further employ the technique of vector calculations to predict and for see their future path. Finally, he will investigate previous years' data on the butterfly numbers and location to formulate a likely error margin for his prediction. At every step of this process, the biologist has to focus on mathematics to gauge, predict, analyze and understand natural phenomena.

\section{Mathematical Model}

In this chapter, we proposed a fractional order SVIR epidemic model is incorporated to investigate its dynamical behavior in random environments. S, V, I, R are known as variables and these variables represent the number of susceptible, vaccinated,infected and recovered cells from viruses in the body. The Caputo fractional derivative operator of order $\alpha \epsilon(0,1]$ is employed to obtain the system of fractional differential equations. The basic reproductive number is derived for a general viral production rate which determines the local stability of the infection free equilibrium. The stability and sensitivity analysis of fractional order has been made and verify the non-negative unique solution.The solution of the time fractional model has been procured by employing Laplace Adomian decomposition method (LADM) and the accuracy of the scheme is presented by convergence analysis. Finally numerical solutions are also established to investigate the influence of system parameter on the spread of disease and which show the effect of fractional parameter on our obtained solution.

Thus, the mathematical model is represented by the following four differentials equations: This model is the basic compartmental model in epidemiology. Since then,many compartmental models such as SIR or SIRS models have been established to predict the various properties of the diseases spreading.Hence vaccination can also be considered by adding some compartment naturally into the basic epidemic models 
for certain diseases.

In formulation of SVIR epidamic model, Here we uses four different variables here S represents the compartment of susceptible cells (i.e., the compartment of those individuals which are not infected an able to catch the disease), V compartment represents the vaccinated cells, I compartment represents the already infected cells and $\mathrm{R}$ represents the recovered cells from viruses in the body. This model has the following form:

$$
\begin{array}{r}
\frac{d S}{d t}=\mu-\mu S-\beta S I-\alpha S, \\
\frac{d V}{d t}=\alpha S-\beta_{1} V I-\gamma_{1} V-\mu V, \\
\frac{d I}{d t}=\beta S I+\beta_{1} V I-\gamma I-\mu I, \\
\frac{d R}{d t}=\gamma_{1} V+\gamma I-\mu R .
\end{array}
$$

Here $\mathrm{S}(\mathrm{t}), \mathrm{I}(\mathrm{t})$ and $\mathrm{R}(\mathrm{t})$ denote the densities of susceptible, infected and recovered individuals, respectively. $\mathrm{V}(\mathrm{t})$ denotes the density of vaccinees who have begun the vaccination prodess.From [12] it follows that all the parameters in the model are positive and have the following features, $\mu$ is the recruitment rate and natural death rate of the population, $\beta$ denotes the transmission coefficient between compartments $\mathrm{S}$ and $\mathrm{I}$, $\alpha$ is the rate at which susceptible individuals are moved into the vaccination process, $\beta_{1}$ denotes the disease transmission rate when the vaccinees contact with infected individuals before obtaining immunity, $\gamma_{1}$ is the average rate for the vaccinees move into recovered population, $\gamma$ is the recovery rate of infected individuals. We assume that $\beta_{1}$ is less than $\beta$ because the vaccinating may have some partial immunity during the process. The fractional order extension for the ordinary differential equations of the model have been first studied in $[13,14]$. The new system of differential equation is represented by the fractional system of differential equations (FDEs) is given as follows.

$$
\begin{array}{r}
D^{\alpha_{1}}(S)=\mu-\mu S-\beta S I-\alpha I \\
D^{\alpha_{2}}(V)=\alpha S-\beta_{1} V I-\lambda_{1} V-\mu V \\
D^{\alpha_{3}}(I)=\beta S I+\beta_{1} V I-\gamma I-\mu I \\
D^{\alpha_{4}}(R)=\lambda_{1} V+\lambda I-\mu R
\end{array}
$$

with the initial conditions

$$
S(0)=n_{1}=0.4, V(0)=n_{2}=0.4, I(0)=n_{3}=0.2, R(0)=n_{4}=0.1
$$




\section{Qualitative Analysis}

To evaluate the equilibrium point, we take

$D^{\alpha_{1}}(S)=0, D^{\alpha_{2}}(V)=0, D^{\alpha_{3}}(I)=0, D^{\alpha_{4}}(R)=0$

above model becomes

$$
\begin{aligned}
\mu-\mu S-\beta S I-\alpha S & =0 \\
\alpha S-\beta_{1} V I-\gamma_{1} V-\mu V & =0 \\
\beta S I+\beta_{1} V I-\gamma I-\mu I & =0 \\
\gamma_{1} V+\gamma I-\mu R & =0
\end{aligned}
$$

After simplifications (2.1),(2.2),(2.3) and (2.4) we get

$S=\frac{\mu}{\alpha+\mu}, V=\frac{\alpha \mu}{(\alpha+\mu)\left(\mu+\gamma_{1}\right)}, I=0, R=\frac{\alpha \gamma_{1}}{(\alpha+\mu)\left(\mu+\gamma_{1}\right)}$

after parametric values

$\mu=1, \beta=15, \alpha=15, \beta_{1}=10, \gamma=2, \gamma_{1}=4$

we get

$(S, V, I, R)=(0.0625,0.1875,0,0.75)$

3.1. Reproductive Number. Consider the jacobian matrix to find the reproductive number by using next generation technique

$$
J=\left[\begin{array}{cccc}
-\mu-\beta I-\alpha & 0 & -\beta S & 0 \\
\alpha & \beta_{1} I-\gamma_{1}-\mu & \beta_{1} V & 0 \\
\beta I & \beta_{1} I & \beta S+\beta_{1} V-\gamma-\mu & 0 \\
0 & \gamma_{1} & \gamma & -\mu
\end{array}\right] .
$$

we take $\mathrm{J}=\mathrm{F}-\mathrm{V}$,

where

$$
F=\left[\begin{array}{cccc}
0 & 0 & 0 & 0 \\
0 & \beta_{1} I & \beta_{1} V & 0 \\
0 & \beta_{1} I & \beta_{1} V & 0 \\
0 & 0 & 0 & 0
\end{array}\right]
$$


and

$$
V=\left[\begin{array}{cccc}
\mu+\beta I+\alpha & 0 & +\beta S & 0 \\
-\alpha & \gamma_{1}+\mu & 0 & 0 \\
-\beta I & 0 & -\beta S+\gamma+\mu & 0 \\
0 & -\gamma_{1} & -\gamma & \mu
\end{array}\right]
$$

By using the relation $|K-\lambda I|=0$, where $K=F V^{-1}$ and $S=\frac{\mu}{\alpha+\mu}, V=\frac{\alpha \mu}{(\alpha+\mu)\left(\mu+\gamma_{1}\right)}$ and $\mathrm{I}=0$

we get eigen value

$$
\lambda=\frac{\alpha \mu \beta_{1}\left(\mu+\gamma_{1}\right)}{\alpha(\gamma+\mu)+\mu(-\beta+\gamma+\mu)}
$$

which represents the reproductive number

$$
R_{0}=\frac{\alpha \mu \beta_{1}\left(\mu+\gamma_{1}\right)}{\alpha(\gamma+\mu)+\mu(-\beta+\gamma+\mu)}
$$

Which is

$$
R_{0}=1.2>1
$$

\section{Stability Analysis}

Theorem 4.1. The endemic equilibrium state $E_{1}=(S, V, I, R)$ of the model (1.1)-(1.4) is locally asymptotically stable, if $R_{0}>1$, otherwise unstable.

To prove this theorem, we have to show that the $\operatorname{Re}(\lambda)<0$. Consider the jacobian matrix as

$$
J=\left[\begin{array}{cccc}
-\mu-\beta I-\alpha & 0 & -\beta S & 0 \\
\alpha & -\beta_{1} I-\gamma_{1}-\mu & -\beta_{1} V & 0 \\
\beta I & \beta_{1} I & \beta S+\beta_{1} V-\gamma-\mu & 0 \\
0 & \gamma_{1} & \gamma & -\mu
\end{array}\right]
$$


Take $\mathrm{I}=0$ (As infected component), we get

$$
J_{0}=\left[\begin{array}{cccc}
-\mu-\alpha & 0 & -\beta S & 0 \\
\alpha & -\gamma_{1}-\mu & -\beta_{1} V & 0 \\
0 & 0 & \beta S+\beta_{1} V-\gamma-\mu & 0 \\
0 & \gamma_{1} & \gamma & -\mu
\end{array}\right]
$$

By using the relation $\left|J_{0}-\lambda I\right|=0$ we get the result

$\lambda_{1}=-\alpha-\mu<0$

$\lambda_{2}=-\mu<0$

$\lambda_{3}=-\mu-\gamma_{1}<0$

$\lambda_{4}=-\gamma-\mu+\frac{\beta \mu}{\alpha+\mu}+\frac{\beta_{1} \alpha \mu}{(\alpha+\mu)\left(\mu+\gamma_{1}\right)}<0$

All the real values of $\lambda$ are negative. So,our system for endemic equilibrium is locally asymptotically stable.

\section{Preliminaries}

In this section, we give some fundamental results and definitions from fractional calculus. For detailed over view of the topic readers are referred to $[15,16]$.

Definition 4.1 The Riemann-liouville fractional integration of order $\alpha \in(0,1)$ of the function $f \in L^{1}([0, T], R)$ is defined as as

$$
I_{0+}^{\alpha} f(t)=\frac{1}{\Gamma(\alpha)} \int_{0}^{t}(t-s)^{\alpha-1} f(s) d s
$$

The Riemann-Liouville derivative has certain disadvantages such that the fractional derivative of a constant is not zero. Therefore, we will make use of Caputo's definition owing to its convenience for initial conditions of the fractional differential equations.

Definition 4.2 The definitions of Laplace transform of Caputo's derivative is written as

$$
L\left\{{ }^{c} D^{\alpha} y(t)\right\}=s^{\alpha} h(s)-\Sigma_{k=0}^{n-1} s^{\alpha-i-1} y^{(k)}(0), n-1<\alpha \leq n ;{ }^{\natural} n \in N .
$$

for arbitrary $c_{i} \in R, i=0,1,2, \ldots, n-1$, where $n=[\alpha]+1$ and $[\alpha]$ shows the integer part of $\alpha$.

\section{The Laplace-Adomian Decomposition Method}

Consider the fractional-order epidemic model (1.5)-(1.8) subject to the initial condition (1.9). The nonlinear term in this model is $S I$ and VI For $\phi_{1}=\phi_{2}=\phi_{3}=\phi_{4}=\phi_{5}=1$ the fractional order model converts 
to the classical epidemic model. by using Laplace transform on the system given in equations (1.5)-(1.8), we get

$$
\begin{gathered}
\mathscr{L}\left\{D_{t}^{\alpha_{1}} S\right\}=\mu \mathscr{L}\{1\}-\mu \mathscr{L}\{S\}-\beta \mathscr{L}\{S I\}-\alpha \mathscr{L}\{S\} \\
\mathscr{L}\left\{D_{t}^{\alpha_{2}} V\right\}=\alpha \mathscr{L}\{S\}-\beta_{1} \mathscr{L}\{V I\}-\lambda_{1} \mathscr{L}\{V\}-\mu \mathscr{L}\{V\} \\
\mathscr{L}\left\{D_{t}^{\alpha_{3}} I\right\}=\beta \mathscr{L}\{S I\}-\beta_{1} \mathscr{L}\{V I\}-\gamma \mathscr{L}\{I\}-\mu \mathscr{L}\{I\} \\
\mathscr{L}\left\{D_{t}^{\alpha_{4}} R\right\}=\lambda_{1} \mathscr{L}\{V\}-\lambda \mathscr{L}\{I\}-\mu \mathscr{L}\{R\}
\end{gathered}
$$

By the defination laplace transform, we get

$$
\begin{gathered}
S^{\alpha_{1}} \mathscr{L}\{S\}-S^{\alpha_{1}-1} S(0)=\mu \mathscr{L}\{1\}-\mu \mathscr{L}\{S\}-\beta \mathscr{L}\{S I\}-\alpha \mathscr{L}\{S\} \\
S^{\alpha_{2}} \mathscr{L}\{V\}-S^{\alpha_{2}-1} V(0)=\alpha \mathscr{L}\{S\}-\beta_{1} \mathscr{L}\{V I\}-\lambda_{1} \mathscr{L}\{V\}-\mu \mathscr{L}\{V\} \\
S^{\alpha_{3}} \mathscr{L}\{I\}-S^{\alpha_{3}-1} I(0)=\beta \mathscr{L}\{S I\}-\beta_{1} \mathscr{L}\{V I\}-\gamma \mathscr{L}\{I\}-\mu \mathscr{L}\{I\} \\
S^{\alpha_{4}} \mathscr{L}\{R\}-S^{\alpha_{4}-1} R(0)=\lambda_{1} \mathscr{L}\{V\}-\lambda \mathscr{L}\{I\}-\mu \mathscr{L}\{R\}
\end{gathered}
$$

By using the initial condition

$$
\begin{gathered}
\mathscr{L}\{S\}=\frac{n_{1}}{S}+\frac{\mu}{S^{\alpha_{1}+1}}-\frac{\mu}{S^{\alpha_{1}}} \mathscr{L}\{S\}-\frac{\beta}{S^{\alpha_{1}}} \mathscr{L}\{S I\}-\frac{\alpha}{S^{\alpha_{1}}} \mathscr{L}\{S\} \\
\mathscr{L}\{V\}=\frac{n_{2}}{S}+\frac{\alpha}{S^{\alpha_{2}}} \mathscr{L}\{S\}-\frac{\beta_{1}}{S^{\alpha_{2}}} \mathscr{L}\{V I\}-\frac{\lambda_{1}}{S^{\alpha_{2}}} \mathscr{L}\{V\}-\frac{\mu}{S^{\alpha_{2}}} \mathscr{L}\{V\} \\
\mathscr{L}\{I\}=\frac{n_{3}}{S}+\frac{\beta}{S^{\alpha_{3}}} \mathscr{L}\{S I\}-\frac{\beta_{1}}{S^{\alpha_{3}}} \mathscr{L}\{V I\}-\frac{\gamma}{S^{\alpha_{3}}} \mathscr{L}\{I\}-\frac{\mu}{S^{\alpha_{3}}} \mathscr{L}\{I\} \\
\mathscr{L}\{R\}=\frac{n_{4}}{S}+\frac{\lambda_{1}}{S^{\alpha_{4}}} \mathscr{L}\{V\}+\frac{\lambda}{S^{\alpha_{4}}} \mathscr{L}\{I\}-\frac{\mu}{S^{\alpha_{4}}} \mathscr{L}\{R\}
\end{gathered}
$$

It should be assumed that method gives the solution as an infinite series

$$
S=\sum_{k=0}^{\infty} S_{k}, V=\sum_{k=0}^{\infty} V_{k}, I=\sum_{k=0}^{\infty} I_{k}, R=\sum_{k=0}^{\infty} R_{k}
$$

The nonlinearity $S I$ and $V I$ can be written as

$$
I=\sum_{k=0}^{\infty} A_{k}, V I=\sum_{k=0}^{\infty} B_{k}
$$


where $A_{k}$ and $B_{k}$ is called the Adomian polynomials given as

$$
\begin{aligned}
& A_{k}=\left.\frac{1}{k !} \frac{d^{k}}{d \lambda^{k}}\left[\sum_{j=0}^{k} \lambda^{j} S_{j} \sum_{j=0}^{k} \lambda^{j} I_{j}\right]\right|_{\lambda=0} \\
& B_{k}=\left.\frac{1}{k !} \frac{d^{k}}{d \lambda^{k}}\left[\sum_{j=0}^{k} \lambda^{j} V_{j} \sum_{j=0}^{k} \lambda^{j} I_{j}\right]\right|_{\lambda=0}
\end{aligned}
$$

From equations 3.1, 3.2, 3.3 and 3.4, we have the followings results

$$
\mathscr{L}\left\{S_{0}\right\}=\frac{n_{1}}{S}+\frac{\mu}{S^{\alpha_{1}+1}}, \mathscr{L}\left\{V_{0}\right\}=\frac{n_{2}}{S}, \mathscr{L}\left\{I_{0}\right\}=\frac{n_{3}}{S}, \mathscr{L}\left\{R_{0}\right\}=\frac{n_{4}}{S}
$$

similarly, we have

$$
\begin{aligned}
& \mathscr{L}\left\{S_{1}\right\}=-\frac{\mu}{S^{\alpha_{1}}} \mathscr{L}\left\{S_{0}\right\}-\frac{\beta}{S^{\alpha_{1}}} \mathscr{L}\left\{A_{0}\right\}-\frac{\alpha}{S^{\alpha_{1}}} \mathscr{L}\left\{S_{0}\right\} \\
& \mathscr{L}\left\{S_{k+1}\right\}=-\frac{\mu}{S^{\alpha_{1}}} \mathscr{L}\left\{S_{k}\right\}-\frac{\beta}{S^{\alpha_{1}}} \mathscr{L}\left\{A_{k}\right\}-\frac{\alpha}{S^{\alpha_{1}}} \mathscr{L}\left\{S_{k}\right\} \\
& \mathscr{L}\left\{V_{1}\right\}=\frac{\alpha}{S^{\alpha_{2}}} \mathscr{L}\left\{S_{0}\right\}-\frac{\beta_{1}}{S^{\alpha_{2}}} \mathscr{L}\left\{B_{0}\right\}-\frac{\lambda_{1}}{S^{\alpha_{2}}} \mathscr{L}\left\{V_{0}\right\}-\frac{\mu}{S^{\alpha_{2}}} \mathscr{L}\left\{V_{0}\right\} \\
& \mathscr{L}\left\{V_{k+1}\right\}=\frac{\alpha}{S^{\alpha_{2}}} \mathscr{L}\left\{S_{k}\right\}-\frac{\beta_{1}}{S^{\alpha_{2}}} \mathscr{L}\left\{B_{k}\right\}-\frac{\lambda_{1}}{S^{\alpha_{2}}} \mathscr{L}\left\{V_{k}\right\}-\frac{\mu}{S^{\alpha_{2}}} \mathscr{L}\left\{V_{k}\right\} \\
& \mathscr{L}\left\{I_{1}\right\}=\frac{\beta}{S^{\alpha_{3}}} \mathscr{L}\left\{A_{0}\right\}+\frac{\beta_{1}}{S^{\alpha_{3}}} \mathscr{L}\left\{B_{0}\right\}-\frac{\gamma}{S^{\alpha_{3}}} \mathscr{L}\left\{I_{0}\right\}-\frac{\mu}{S^{\alpha_{3}}} \mathscr{L}\left\{I_{0}\right\} \\
& \mathscr{L}\left\{I_{k+1}\right\}=\frac{\beta}{S^{\alpha_{3}}} \mathscr{L}\left\{A_{k}\right\}+\frac{\beta_{1}}{S^{\alpha_{3}}} \mathscr{L}\left\{B_{k}\right\}-\frac{\gamma}{S^{\alpha_{3}}} \mathscr{L}\left\{I_{k}\right\}-\frac{\mu}{S^{\alpha_{3}}} \mathscr{L}\left\{I_{k}\right\}
\end{aligned}
$$




$$
\begin{gathered}
\mathscr{L}\left\{R_{1}\right\}=\frac{\lambda_{1}}{S^{\alpha_{4}}} \mathscr{L}\left\{V_{0}\right\}+\frac{\lambda}{S^{\alpha_{4}}} \mathscr{L}\left\{I_{0}\right\}-\frac{\mu}{S^{\alpha_{4}}} \mathscr{L}\left\{R_{0}\right\} \\
\cdot \\
\cdot \\
\mathscr{L}\left\{R_{k+1}\right\}=\frac{\lambda_{1}}{S^{\alpha_{4}}} \mathscr{L}\left\{V_{k}\right\}+\frac{\lambda}{S^{\alpha_{4}}} \mathscr{L}\left\{I_{k}\right\}-\frac{\mu}{S^{\alpha_{4}}} \mathscr{L}\left\{R_{k}\right\}
\end{gathered}
$$

The purpose of the work is to analysis the mathematical behavior of the solution $S(t), V(t), I(t), R(t)$ for the different values of $\phi$. By applying the inverse laplace transform to both sides of the equation (3.9), we get the values of $S_{0}, V_{0}, I_{0}, R_{0}$ and used for further process. Putting the values of $S_{0}, V_{0}, I_{0}, R_{0}$ and $A_{0}, B_{0}$ into the equations (3.12-3.16) and get the values of $S_{1}, V_{1}, I_{1}, R_{1}$. Similarly we find the remaining term $S_{2}, S_{3}, S_{4}, \ldots, V_{2}, V_{3}, V_{4}, \ldots, I_{2}, I_{3}, I_{4}, \ldots, R_{2}, R_{3}, R_{4}, \ldots$ and in the same manners. We have the series solution in the form

$$
\begin{array}{r}
S(t)=S_{0}+S_{1}+S_{2}+S_{3}+S_{4}+\ldots \\
V(t)=V_{0}+V_{1}+V_{2}+V_{3}+V_{4}+\ldots \\
I(t)=I_{0}+I_{1}+I_{2}+I_{3}+I_{4}+\ldots \\
R(t)=R_{0}+R_{1}+R_{2}+R_{3}+R_{4}+\ldots .
\end{array}
$$

The Laplace Adomian decomposition method is an analytical approximate solution in terms of infinite power series. For numerical results, we used the followings table values of parameters are considered from

TABLE 1. Values of physical parameters used in SVIR model when $R_{0}>1$

\begin{tabular}{|c|c|c|c|}
\hline Parameter & Value & Parameter & Value \\
\hline$\alpha$ & 15 & $\beta$ & 15 \\
\hline$\gamma$ & 2 & $\mu$ & 1 \\
\hline$\beta_{1}$ & 10 & $\gamma_{1}$ & 4 \\
\hline
\end{tabular}

By applying Laplace inverse property on equation (3.18-3.21)as describes above, we can find the solution of fractional model in series form is written as

$$
\begin{aligned}
S(t)=0.4-6.6 \frac{t^{\alpha_{1}}}{\alpha_{1} !} & +125.4 \frac{t^{2 \alpha_{1}}}{2 \alpha_{1} !}+361 \frac{t^{3 \alpha_{1}}}{3 \alpha_{1} !}-9 \frac{t^{\alpha_{1}+\alpha_{3}}}{\left(\alpha_{1}+\alpha_{3}\right) !}-18 \frac{t^{2 \alpha_{1}+\alpha_{3}}}{\left(2 \alpha_{1}+\alpha_{3}\right) !} \\
- & 21 \frac{\left(\alpha_{1}+\alpha_{3}\right) ! t^{2 \alpha_{1}+\alpha_{3}}}{\alpha_{1} \alpha_{3}\left(2 \alpha_{1}+\alpha_{3}\right) !}-45 \frac{\left(2 \alpha_{1}+\alpha_{3}\right) ! t^{3 \alpha_{1}+\alpha_{3}}}{\alpha_{1} !\left(\alpha_{1}+\alpha_{3}\right) !\left(3 \alpha_{1}+\alpha_{3}\right) !}+\ldots .
\end{aligned}
$$




$$
\begin{gathered}
V(t)=0.4+3.2 \frac{t^{\alpha_{2}}}{\alpha_{2} !}-99 \frac{t^{\alpha_{1}+\alpha_{2}}}{\left(\alpha_{1}+\alpha_{2}\right) !}-285 \frac{t^{2 \alpha_{1}+\alpha_{2}}}{\left(2 \alpha_{1}+\alpha_{2}\right) !}-6 \frac{t^{\alpha_{2}+\alpha_{3}}}{\left(\alpha_{2}+\alpha_{3}\right) !}, \\
-12 \frac{t^{\alpha_{1}+\alpha_{2}+\alpha_{3}}}{\left(\alpha_{1}+\alpha_{2}+\alpha_{3}\right) !}-22.4 \frac{t^{2 \alpha_{2}}}{2 \alpha_{2} !}-105 \frac{t^{\alpha_{1}+2 \alpha_{2}}}{\left(\alpha_{1}+2 \alpha_{2}\right) !}+\ldots \ldots \\
I(t)=0.2+1.5 \frac{t^{\alpha_{3}}}{\alpha_{3} !}+3 \frac{t^{\alpha_{1}+\alpha_{3}}}{\left(\alpha_{1}+\alpha_{3}\right) !}+91.8 \frac{t^{2 \alpha_{3}}}{2 \alpha_{3} !}+21 \frac{t^{\alpha_{1}+2 \alpha_{3}}}{\left(\alpha_{1}+2 \alpha_{3}\right) !}+21 \frac{\left(\alpha_{1}+\alpha_{3}\right) ! t^{\alpha_{1}+2 \alpha_{3}}}{\alpha_{1} ! \alpha_{3} !\left(\alpha_{1}+2 \alpha_{3}\right) !}, \\
+45 \frac{\left(2 \alpha_{1}+\alpha_{3}\right) ! t^{\alpha_{1}+2 \alpha_{3}}}{\alpha_{1} !\left(\alpha_{1}+\alpha_{3}\right) !\left(\alpha_{1}+2 \alpha_{3}\right) !}-22.8 \frac{t^{\alpha_{1}}+\alpha_{3}}{\left(\alpha_{1}+\alpha_{3}\right) !}-57 \frac{t^{2 \alpha_{1}+\alpha_{3}}}{\left(2 \alpha_{1}+\alpha_{3}\right) !}+6.4 \frac{t^{\alpha_{2}+\alpha_{3}}}{\left(\alpha_{2}+\alpha_{3}\right) !} \\
R(t)=0.1+1.9 \frac{t^{\alpha_{4}}}{\alpha_{4} !}+12.8 \frac{t^{\alpha_{2}+\alpha_{4}}}{\left(\alpha_{2}+\alpha_{4}\right) !}+60 \frac{t^{\alpha_{1}+\alpha_{2}+\alpha_{3}}}{\left(\alpha_{1}+\alpha_{2}+\alpha_{2}+\alpha_{4}\right) !}+2.28 \frac{\left.t^{\alpha_{3}+\alpha_{4}}+\alpha_{3}\right) !}{\left(\alpha_{3}+\alpha_{4}\right) !}, \\
+6 \frac{t^{\alpha_{1}+\alpha_{3}+\alpha_{4}}}{\left(\alpha_{1}+\alpha_{3}+\alpha_{4}\right) !}-1.9 \frac{t^{2 \alpha_{4}}}{2 \alpha_{4} !}+\ldots . .
\end{gathered}
$$

\section{Numerical Results And Discussion}

The mathematical analysis of SVIR epidemic model with nonlinear system of fractional differential equation has been presented. The numerical results of susceptible, Vaccinated, infected and recovered population for different values of $\alpha$ are established in tables 2-5 by using LADM. For the reliable investigation, evaluation is made for different values of $\alpha$ and can be seen from Figure 1-4. we observe that fractional order SVIR epidamic model has more degree of freedom as compared to ordinary derivatives. By taking non-integer values of fractional parameter, remarkable responses of the compartments of the proposed model are obtained. For different values of $\alpha$ solution converges to steady state and gives the better convergence by decreasing the fractional values of $\alpha$. 


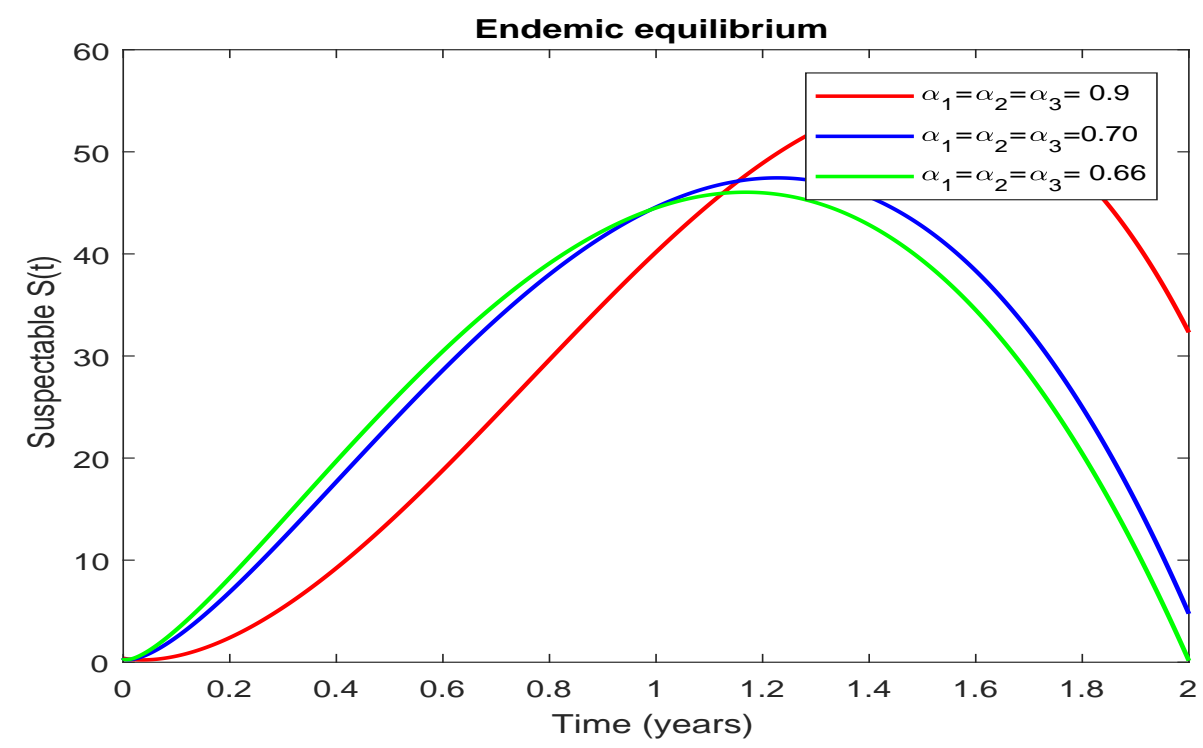

Figure 1. Numerical Solutions for susceptible $S(t)$ population in a time $t$ (months) at different values of $\alpha$.

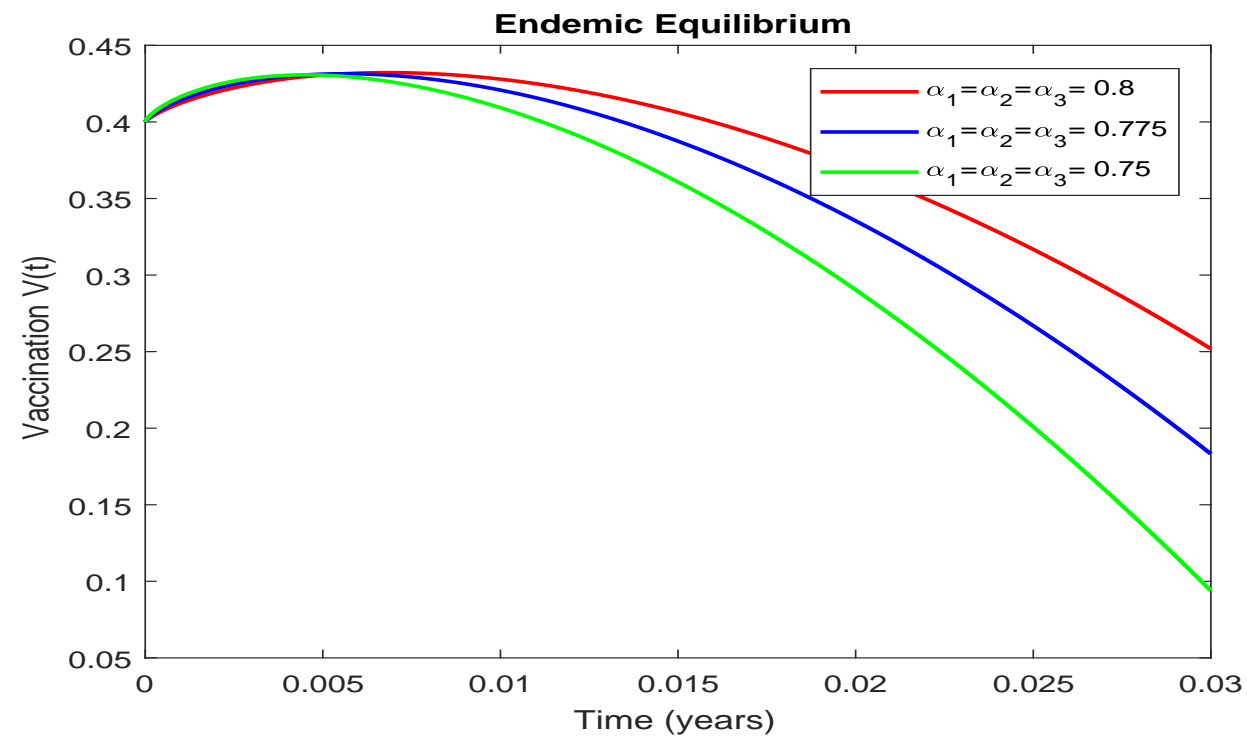

Figure 2. Numerical Solutions for vaccination $V(t)$ population in a time $t$ (months) at different values of $\alpha$. 


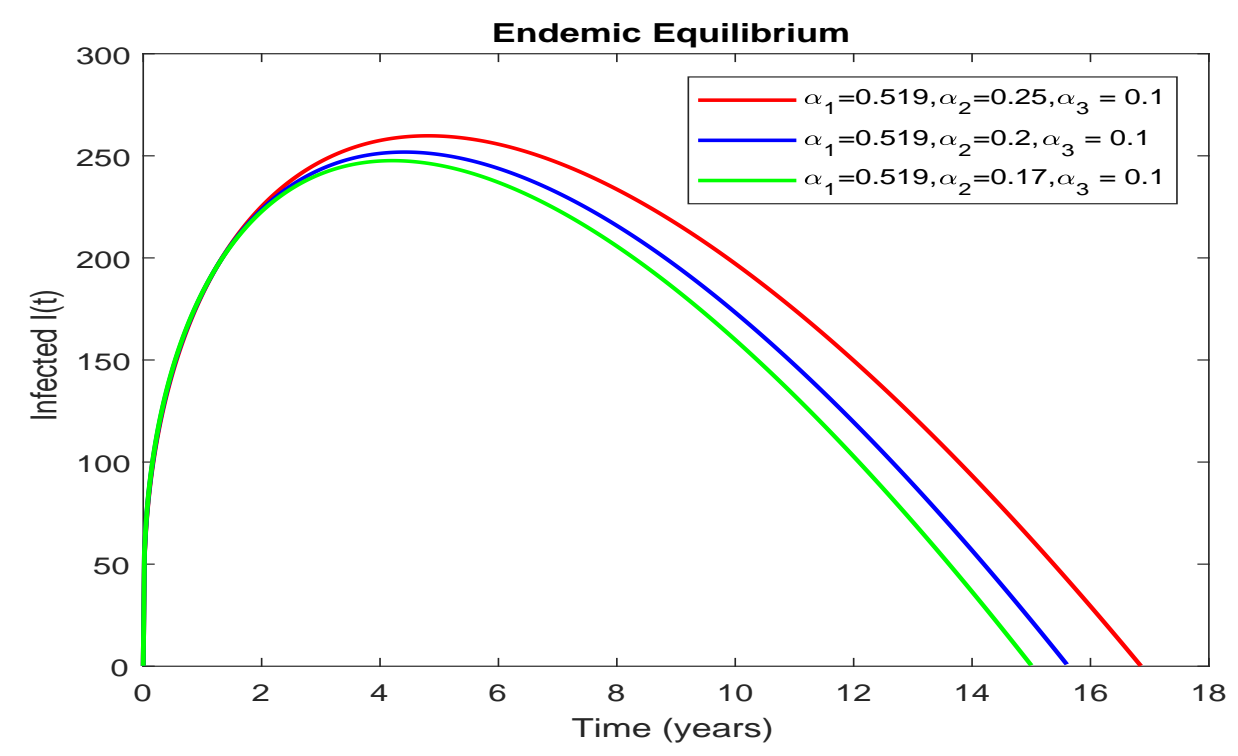

Figure 3. Numerical Solutions for infected $I(t)$ population in a time $t$ (months) at different values of $\alpha$.

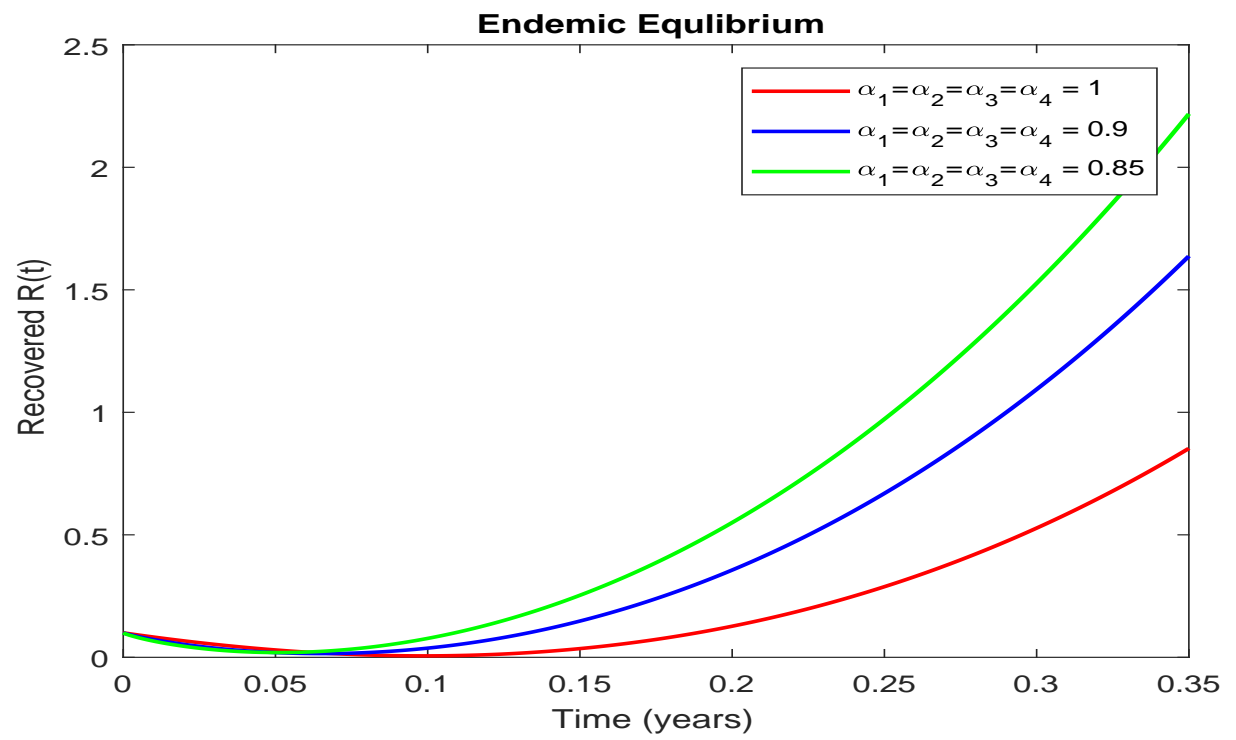

FiguRE 4. Numerical Solutions for recovered $R(t)$ population in a time $t$ (months) at different values of $\alpha$.

\section{Conclusion}

It is important to note that Laplace adomian decomposition method for mathematical models based on system of fractional order differential equations is more powerful approach to compute the convergent solutions.We developed a scheme for analytical solution of epidemic fractional SVIR model by using Laplace 
Adomian decomposition method. The well-known epidemic model namely Susceptible-Vaccination-InfectedRecovered (SVIR) is considered with and without demographic effects. The model represents population dynamics during the disease as a set of non-linear coupled ordinary differential equations. There is no exact solution available in the literature for this model up to the best of authors knowledge. It is observed that the infection rate and reproductive numbers play a key role for an epidemic to occur and the epidemic can be controlled by vaccination. It is also observed that to eliminate the disease, it is not necessary to vaccinate whole of the population.The effect of fractional parameter on our obtained solutions is presented through Tables and graphs. It is worthy to observe that fractional derivatives show significant changes and memory effects as compared to ordinary derivatives. Finally, we estimated the parameter that characterize the behavior of disease and present numerical simulations. The disease free equilibrium state is carried out by presenting $\operatorname{Re}(\lambda)<0$, i.e. the population is viable.

\section{REFERENCES}

[1] J. Biazar, Solution of the epidemic model by Adomian decomposition method, Appl. Math. Comput. 173 (2006), $1101-1106$.

[2] S. Busenberg, P. Driessche, Analysis of a disease transmission model in a population with varying size, J. Math. Biol. 28 (1990), 65-82.

[3] AE A.M.A. El-Sayed, S.Z. Rida, A.A.M. Arafa, On the solutions of time-fractional bacterial chemotaxis in a diffusion gradient chamber, Int. J. Nonlinear Sci. 7 (2009), 485-495.

[4] O.D. Makinde, Adomian decomposition approach to a SIR epidemic model with constant vaccination strategy, Appl. Math. Comput. 184 (2007), 842-848.

[5] A.A.M. Arafa, S.Z. Rida, M. Khalil, Fractional modeling dynamics of HIV and 4 T-cells during primary infection, Nonlinear Biomed. Phys. 6 (2012), 1-7.

[6] C.M. Kribs-Zaleta, Structured models for heterosexual disease transmission, Math. Biosci. 160 (1999), 83-108.

[7] B. Buonomo, D. Lacitignola, On the dynamics of an SEIR epidemic model with a convex incidence rate, Ricerche Mat. 57 (2008), 261-281.

[8] X. Liu, C. Wang, Bifurcation of a predator-prey model with disease in the prey, Nonlinear Dyn. 62 (2010), 841-850.

[9] Fazal Haq, Kamal Shah, Ghaus ur Rahman, Muhammad Shahzad. Numerical solution of fractional order smoking model via laplace Adomian decomposition method, Alex. Eng. J. 57 (2) (2018), 1061-1069

[10] J.D Murray: Mathematical Biology I. An Introduction,USA ,Springer, Verlag Berlin Heidelberg. (2002).

[11] A. C. Guyton and J. E. Hall, Text Book of Medical Physiology, Elsevier Saunders, St. Louis, edition 11972 (2012).

[12] Erturk, VS, Zaman, G, Momani, S: A numeric analytic method for approximating a giving up smoking model containing fractional derivatives. Comput. Math. Appl. 64 (2012), 3068-3074.

[13] Zeb, A, Chohan, I, Zaman, G: The homotopy analysis method for approximating of giving up smoking model in fractional order. Appl. Math. 3 (2012), 914-919.

[14] Alkhudhari, Z, Al-Sheikh, S, Al-Tuwairqi, S: Global dynamics of a mathematical model on smoking. Appl. Math. 2014 (2014), Article ID 847075.

[15] R. Magin, Fractional Calculus in Bioengineering, Begell House Publishers, 2004.

[16] J. Biazar, Solution of the epidemic model by Adomian decomposition method, Appl. Math. Comput. 173 (2006), 1101-1106. 
[17] T. Khan, G. Zaman and M. I. Chohan, The transmission dynamic and optimal control of acute and chronic hepatitis B, J. Biol. Dyn. 11 (1) (2017), 172-189.

[18] Aqeel Ahmad, Muhammad Farman, Faisal Yasin, M. O. Ahmad, Dynamical transmission and effect of smoking in society, Int. J. Adv. Appl. Sci. 5(2) (2018), 71-75

[19] Aqeel Ahmad, Muhammad Farman, M. O. Ahmad, Nauman Raza, M. Abdullah, Dynamical behavior of SIR epidemic model with non-integer time fractional derivatives: A mathematical analysis, Int. J. Adv. Appl. Sci. 5(1) (2018), 123-129

[20] Farah Ashraf, Aqeel Ahmad, Muhammad Umer Saleem, Muhammad Farman, M. O. Ahmad, Dynamical behavior of HIV immunology model with non-integer time fractional derivatives, Int. J. Adv. Appl. Sci. 5(3) (2018), 39-45 